\title{
The Stellar Complexes in the Large Magellanic Cloud
}

\author{
F. Maragoudaki and M. Kontizas
}

Section of Astrophysics Astronomy \& Mechanics, Department of

Physics, University of Athens, GR-157 83 Athens, Greece

E. Kontizas and A. Dapergolas

Astronomical Institute, National Observatory of Athens, P.O. Box 20048, GR-118 10 Athens, Greece

\section{D.H. Morgan}

Schmidt Telescope Unit, Royal Observatory of Edinburgh, EH9 3HJ, UK

\begin{abstract}
A method has been developed for the detection of stellar complexes and the derivation of their fundamental properties in the LMC. We have used digitized UKST plates in order to perform star counts and produce isodensity contour maps in various colours and luminosity slices. About 50 large stellar groupings have been revealed. Their properties favour a "top-down" mechanism acting in star forming regions.
\end{abstract}

\section{Observational material and detection of stellar complexes}

We use direct photographic plates taken with the UK $1.2 \mathrm{~m}$ Schmidt Telescope, in various wavebands: $\mathrm{U}, \mathrm{R}$ and HeII $(\lambda=4686 \AA)$ down to a magnitude limit of 19-20 mag. The plates were digitized with the fast measuring machines APM and SuperCosmos and the derived data give for the detected images positions, magnitudes etc.

The detection of the stellar complexes is based on:

1. Star counts and isodensity contour maps

Star counts were performed on the U detected stellar images and the isodensity contour map of the field was produced. The regions with enhanced stellar density were considered as large stellar groupings candidates. The local backgrounds were taken into consideration and were subtracted from the studied areas.

2. Luminosity slices

The instrumental magnitudes have been calibrated by us using known CCD magnitudes from the literature (Will et al. 1997). Star counts and isodensity contour maps performed for various luminosities allowed us to study the distribution of stars at the various luminosity slices.

3. Various wavebands

Steps 1 and 2 were performed on U, R and HeII plates. In the U plates, 
the blue stars of the upper main sequence stand out against the older, background stellar populations, while the $\mathrm{R}$ plates cover a wider range of stellar populations. The HeII waveband covers part of the B magnitude and the obscuration by the gas is minimal.

The structures found from the above three steps (larger than $150 \mathrm{pc}$ ) were accepted as stellar complexes under the following criteria:

1. The stellar density of the structure is enhanced by at least $3 \sigma$ above the mean density of the surrounding field in more than two slices in the $U$ plate. At least one of these slices must contain stars brighter than 17.5 mag.

2. The structure is also revealed in the isodensity maps of the HeII data.

3. If a structure is embedded in a larger one its density should be at least $5 \sigma$ above the mean density of the surrounding field.

\section{Discussion}

The above criteria have been applied to a region of the LMC of about $6^{\circ} \times 7^{\circ}$ centered at $\mathrm{RA} \sim 05^{\mathrm{h}} 26^{\mathrm{m}}$ and DEC $\sim-69^{\circ}$.

About 50 large stellar groupings have been revealed showing: (1) hierarchical structure, where the smallest are found within the large ones; (2) their size distribution has peaks at $250 \pm 50 \mathrm{pc}$ (aggregates) and $600 \pm 50 \mathrm{pc}$ (complexes), there are also a few cases with size $1200 \pm 50$ pc (supercomplexes); (3) the luminosity slices show that these structures dissapear at $U \sim 17.5$ mag corresponding to spectral types of about B8 and mass around $3 M_{\odot} ;(4)$ there is evidence that at the fainter magnitudes these structures are aligned to a general trend whereas at the brighter end (B1, O spectral types) they become more clumpy and symmetrical in shape.

This hierarchical picture of stellar groupings and their preferred dimensions which has been also found in other galaxies (M31, M33), favours a "topdown" scenario for the star formation mechanism in these galaxies (Efremov \& Elmegreen 1998).

Acknowledgments. The authors would like to express their sincere thanks to the $1.2 \mathrm{~m}$ U.K Schmidt Telescope Unit for loan of the material, the British Council for financial support and to the General Secretariat of Research and Technology for financial support of this project.

\section{References}

Battinelli, P., Efremov, Yu.N., \& Magnier, E. 1996, A\&A, 314, 51

Efremov, Yu.N. 1995, AJ, 110, 2757

Elmegreen B.G., \& Efremov Yu.N. 1996, ApJ, 466, 802

Kontizas, M., Maravelias, S.E., Kontizas, E., Dapergolas, A., \& Bellas-Velidis I. 1996, A\&A, 308, 40

Will, J.M., Bomans, D.J., \& Dieball, A. 1997, A\&AS, 123, 455 